\title{
Warkany Syndrome Revealed by Recurrent Infections: Case Report
}

\author{
Karima Chraiet Rezgani ${ }^{1 *}$, Rim Abdelkarim ${ }^{2 *}$ \\ ${ }^{1}$ The Faculty of Medicine of Tunis, Tunis El Manar University, Tunisia \\ ${ }^{2}$ Pediatric Department, Mohamed Tlatli Hospital, Nabeul, Tunisia \\ Email: ^chraietkarima@yahoo.fr, ^Rimabdelkrim91@gmail.com
}

How to cite this paper: Chraiet Rezgani, K. and Abdelkarim, R. (2021) Warkany Syndrome Revealed by Recurrent Infections: Case Report. Open Journal of Genetics, 11, 1-8.

https://doi.org/10.4236/ojgen.2021.111001

Received: December 19, 2020

Accepted: March 26, 2021

Published: March 29, 2021

Copyright $\odot 2021$ by author(s) and Scientific Research Publishing Inc. This work is licensed under the Creative Commons Attribution International License (CC BY 4.0).

http://creativecommons.org/licenses/by/4.0/

\begin{abstract}
Introduction: Warkany syndrome, also called trisomy 8 mosaicism (T8M), is a rare genetic abnormality characterized by a large phenotypic variability. This heterogeneity leads to delayed diagnosis in the majority of cases. Frequently, development retardation is the first apparent anomaly that imposes genetic study, and hence diagnosis is done. In other situations, the revealing presentation is atypical. Aims: Report a case of T8M in a child followed up for recurrent respiratory infections and insists on the global assessment of patients. Case Description: The patient, aged six years old, has been followed up since his first year for recurrent infections. At birth, the medical assessment was normal apart from bilateral testis ectopia and hypospadias. By the age of nine months, he presented several respiratory infections associated with wheezing thereafter. Immunity investigations were normal and skin tests were positive for dog hair. By allergen eviction and asthma therapeutics, the infant improved. During his follow-up, development retardation has been noted in addition to facial dysmorphism and limb extremities aberrations. Imaging investigations showed the agenesis of both the corpus callosum and the right kidney. Karyotyping on peripheral leucocytes and fibroblast culture revealed T8M in $6 \%$ and $87 \%$ of examined cells respectively. Conclusion: In the present case, the patient's complaint is related to allergy. However, a global assessment of the child led to a rare condition requiring more care and careful follow-up.
\end{abstract}

\section{Keywords}

Warkany Syndrome, Trisomy 8 Mosaicism, Recurrent Infections, Development Retardation

\section{Introduction}

Warkany syndrome is a genetic disease defined as the presence of three copies of 
chromosome 8 in a clone of organism cells. Though complete trisomy 8 is generally lethal, T8M patients are more likely to survive. Warkany syndrome was first reported in 1971 by Grouchy. His estimated frequency is about 1:25,000 to 1:50,000 births with a male predominance [1] [2].

The syndrome has phenotypic heterogeneity counting from mild to severe mental retardation, craniofacial dysmorphism, cardiovascular and urinary tract anomalies, cryptorchidism, and limb extremities aberrations [1]. This wide clinical variability clarifies the diagnosis delay in most cases [1] [3] [4]. The diagnosis is made by karyotype on peripheral blood cells or better on fibroblasts after skin biopsy.

There is no specific treatment for the disease. However, a multidisciplinary approach can improve the life quality of both the infant and his parents and makes easier patient's social integration later.

\section{Case Report}

The presented case is the second child of a non-consanguineous couple. The parent's origin is Somaa; a village belonging to the Nabeul governorate. Father and mother ages were respectively thirty-four and twenty-nine years old.

The pathological history in the family consists of allergic rhinitis in the mother. The pregnancy was uneventful: neither complication and medication nor radiation to the mother was signed. Plus, her serology screening for TORSCH infections was negative.

The neonate was born at term by cesarean section. Breathing, cardio-circulatory, and neurological assessments were well. Weight and height were respectively equal to three kilograms and forty-nine centimeters. The head circumference at birth was not available. Initially, examination showed bilateral testicular ectopia with hypospadias and thus, the newborn was referred to the pediatric surgeon.

By the age of nine months, the baby was hospitalized for acute fever related to rhinopharyngitis subsequently complicated by purulent otitis. Then, he was readmitted into the pediatric department several times for recurrent ENT infections, bacterial pneumonias, and repeated wheezing. Chest X-ray did not suggest a tracheobronchial tree malformation. The blood cell count did not show either anemia or cytopenia. The immunity study offered normal values of antibodies, both lymphocyte phenotyping and the NBT test (Nitro Blue Tetrazolium test) were normal. Since the mother had an atopic manifestation, our patient was tested for respiratory allergens. The result was positive for dog hair.

The patient's growth had been satisfactory initially. Thereafter, macrocephaly was discovered. The head circumference was at $+2.5 \mathrm{SD}$ at the age of three. Dysmorphism and development retardation became more evident. The child has presented facial dysmorphism with a triangular face, a prominent forehead, hypertelorism, and a broad-based nose as shown in Figure 1. The oral cavity examination has revealed a cleft palate.

The limbs and the skeletal have been also irregular; hands have shown camptodactyly (Figure 2), elbow and wrist motilities have been limited because of 
joint contractures. In addition, the child has had "rocker bottom" feet and prominent plantar furrows as exposed in Figure 3 and Figure 4 respectively.

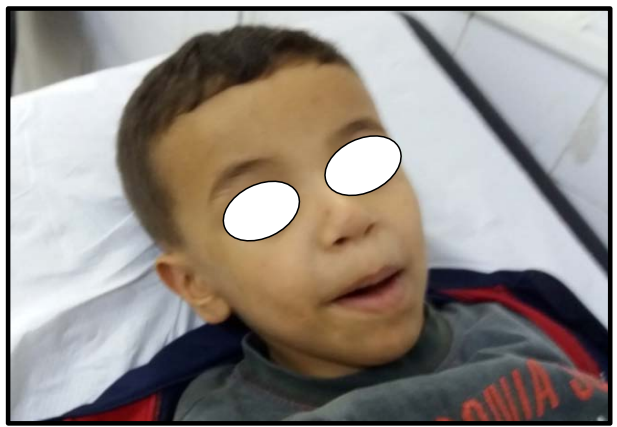

Figure 1. Facial dysmorphism in our patient.

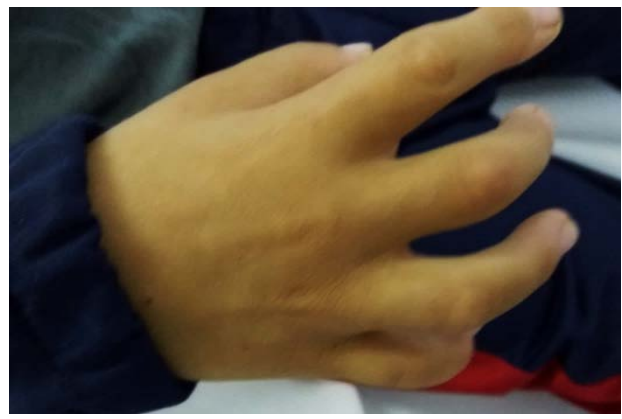

Figure 2. Camptodactyly in the right hand.

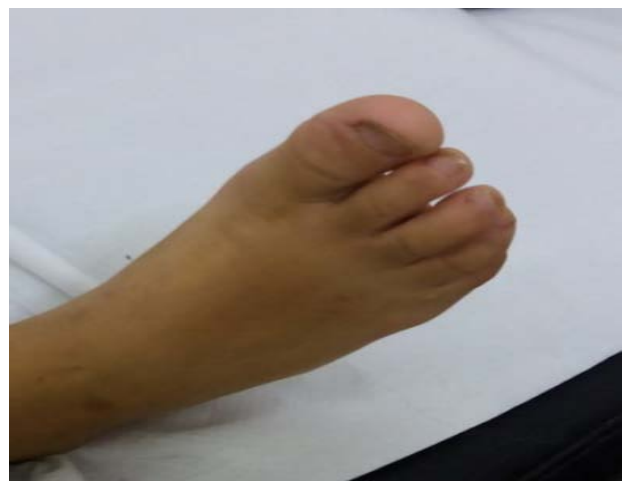

Figure 3. "Rocker bottom" feet.

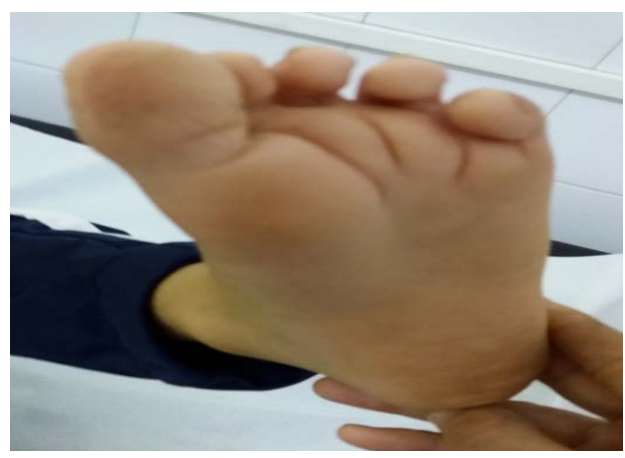

Figure 4. Prominent plantar furrows. 
In addition to the dysmorphism, the infant has had development retardation. He achieved neck holding in the fourth month, independent sitting by the age of one year, and self-reliantly walking after two years. The language was delayed despite normal hearing tests.

The mental retardation became evident at five years old. During medical visits, the child was very close to the parent. He was not attentive neither concentrated. The hand functions were not advanced. The psychological assessment concluded on anxiety attachment disorder without autistic lines.

To identify other organ abnormalities, imaging investigations were done. The cardiac sonography showed minimal aortic regurgitation (Figure 5). Through the abdominal ultrasound, bilateral testis ectopia was confirmed and right kidney agenesis was discovered with hypertrophy of the left one (Figure 6 and Figure 7). Finally, the cerebral MRI concluded on complete agenesis of the corpus callosum.

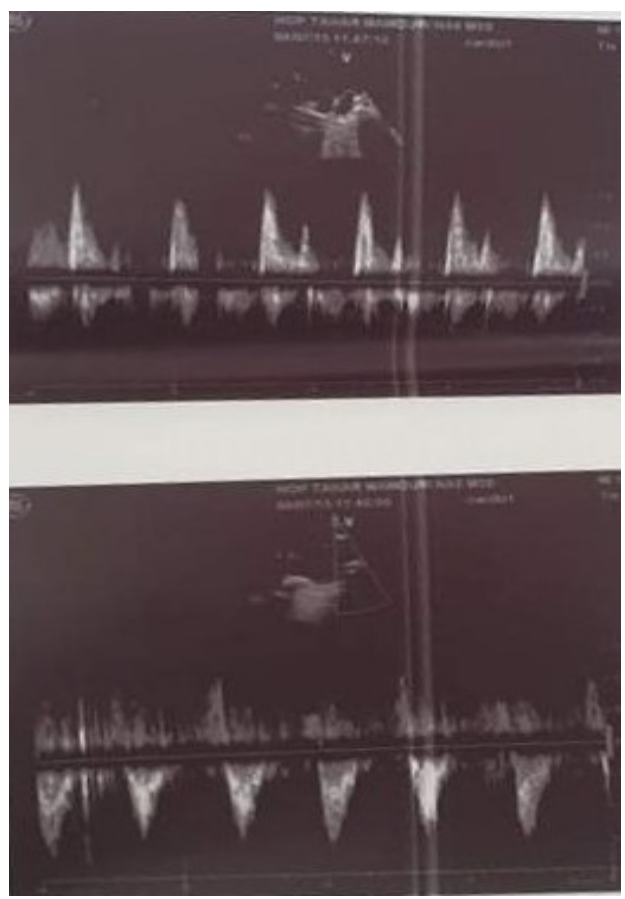

Figure 5. Cardiac sonography showing aortic regurgitation.

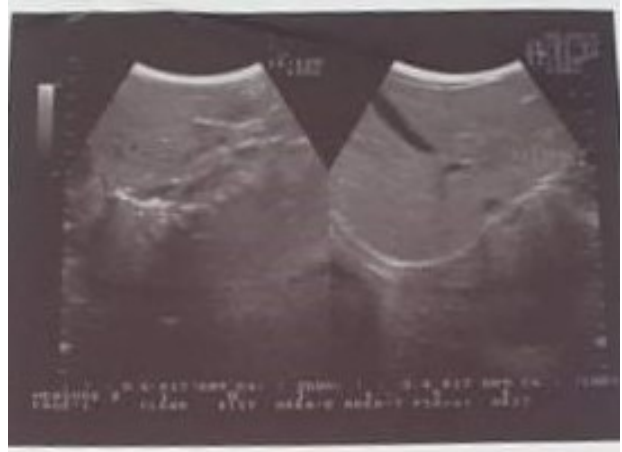

Figure 6. Agenesis of the right kidney. 


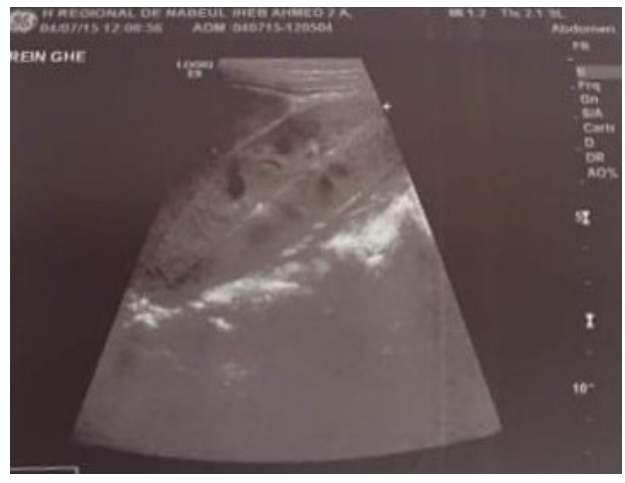

Figure 7. The left kidney.

Given all these anomalies, a karyotype on peripheral leucocytes was performed and revealed T8M $(47, \mathrm{XY},+8 / 46, \mathrm{XY})$ in $6 \%$ of examined cells. The karyotype on fibroblast culture after skin biopsy confirmed T8M in $87 \%$ of cells. The percentage of monosomic cells was equal to 1.6 and that of normal ones was 11.4.

The management was multidisciplinary. By the eviction of the allergen, inhaled corticosteroids, and bronchodilators, respiratory infections and wheezing have improved. The child underwent also surgical intervention for cryptorchidism. After three months of speech therapy, he started pronunciation with few intelligible words. Thus, his integration into a specific school for impaired children was possible. The growth and the routine biological follow-up of the patient have been normal until this date.

\section{Discussion}

Trisomy 8 mosaicism (T8M) is a rare genetic disease and likely underdiagnosed. The origin of this anomaly is most frequently a mitotic non-disjunction during early zygotic development. Maternal meiotic errors may cause total trisomy 8 which generally leads to miscarriages [3]. Despite the wide variability in both cytogenetic and phenotypic expressions, the relation between the degree of mosaicism and clinical features is improbable [5].

Clinical features are heterogeneous but someones seem more likely to be present in a large proportion of patients with T8M. Table 1 shows particularities of clinical presentation in some case reports of literature with comparison to our findings.

As presented in the last table, facial dysmorphism, deep furrows, camptodactyly, skeletal defects, and development retardation are the most frequent features in T8M. In addition to those described above, some other signs were anecdotally reported in papers. We quote as examples infantile spasms, hypothyroidism, and cauda equina syndrome (as a complication of central disk herniation) [4] [6] [7].

Accordingly, in situations of children with major clinical features, T8M must be considered and the karyotyping should not be limited to lymphocyte culture. In fact, the trisomic cell line may disappear from lymphocytes with age; fibroblast 
Table 1. Common clinical features of Trisomy 8 mosaicism in case reports of literature.

\begin{tabular}{|c|c|c|c|c|c|c|}
\hline & & Agrawal A. [3] & Hale N.E. [4] & Udaykumar A.M. [5] & Gustavo G. [6] & Our patient \\
\hline \multicolumn{2}{|c|}{ Age at diagnosis (years) } & 3 & 1.25 & 7 & 14 & 6 \\
\hline \multicolumn{2}{|c|}{ Recurrent infections } & - & + & - & - & + \\
\hline \multicolumn{2}{|c|}{ facial dysmorphism } & + & + & + & + & + \\
\hline \multicolumn{2}{|c|}{ Macrocephaly } & - & + & + & - & + \\
\hline \multicolumn{2}{|c|}{ Camptodactyly } & + & - & + & + & + \\
\hline \multicolumn{2}{|c|}{ Deep palmar and planter furrows } & + & + & + & + & + \\
\hline \multicolumn{2}{|c|}{ Skeletal defects } & + & + & + & + & + \\
\hline \multicolumn{2}{|c|}{ development retardations } & + & + & + & + & + \\
\hline \multicolumn{2}{|c|}{ Deafness } & - & - & + & - & - \\
\hline \multicolumn{2}{|c|}{ Ocular manifestations } & + & + & + & + & - \\
\hline \multicolumn{2}{|c|}{ agenesis of corpus callosum } & - & - & + & - & + \\
\hline \multicolumn{2}{|c|}{ Urogenital tract abnormalities } & - & + & + & - & + \\
\hline \multicolumn{2}{|c|}{ Congenital heart disease } & - & - & - & - & + \\
\hline \multirow{2}{*}{ Percentage of T8 cells } & Blood & $50 \%$ & NM & $18 \%$ & $0 \%$ & $6 \%$ \\
\hline & fibroblasts & NM & NM & NM & $7 \%$ & $87 \%$ \\
\hline
\end{tabular}

NM: not mentioned.

culture becomes crucial for diagnosis [1]. Through this data, the wide difference in the percentage of abnormal cells between the two techniques done in our patient is logical.

Recurrent respiratory infections revealed the T8M in our patient. This manifestation was also reported in few cases of the literature [1] [4] [8]. Hale N.E. and all talked about recurrent otitis media in their patient which was controlled by tonsillectomy and adenoidectomy with insertion of myringotomy tubes. The immunity status of their patient was unknown [4].

On the other hand, Engel B.C. and al described a case of T8M associated with adenosine deaminase deficiency (a kind of severe combined immune deficiency SCID). The patient underwent gene therapy for his congenital immune deficiency after bone marrow cytoreduction. The authors had noted persistent pancytopenia for three months after gene therapy. Bone marrow biopsy was performed and revealed hypocellular marrow and T8M in $21.6 \%$ of cells [8]. Therefore, recurrent infections occurring in Warkany syndrome patients may be related to congenital immune deficiency. In our patient, there was no abnormality in the count of blood cells (CBC) and the immunity investigations were normal.

Repeated CBC in our patient have been reassuring until nowadays. In fact, the surveillance of this exam is necessary for T8M patients even initially normal. Detecting a high level of leucocytes or refractory anemia requires bone marrow study; as malignancy and myeloproliferative disorders are predicted in T8M. Maserati E and all reported a retrospective review of 14 patients who suffered from myelodysplastic syndromes, acute leukemia, or myeloproliferative disorder 
with trisomy 8 . They concluded that in $15 \%$ to $20 \%$ of cases, Trisomy 8 was constitutional [9].

Finally, because of the major handicap related to this syndrome and the possibility of malignancy in this situation, the prenatal assessment for T8M should be done even possible. The cells culture after sampling of chorionic villi, amniotic fluid, or fetal blood allows prenatal diagnosis of this genetic disorder [10] [11]. On the other hand, the results of these investigations and the post-natal outcomes are heterogeneous. Hence, the presence of both fetal ultrasound abnormalities and $\mathrm{T} 8 \mathrm{M}$ in fetal blood sampling has a great contribution to establishing the prognosis of the pregnancy [10] [11].

\section{Conclusion}

Assessment for recurrent infections is common during early childhood. Healthcare providers have to be aware of some rare situations. The case report exposed classic features of Warkany syndrome, known for its wide phenotypic variability. This motivated chromosomal analysis in blood cells and fibroblasts. The confirmation of T8M permitted better healthcare and careful follow-up.

\section{Comments}

The authors have no conflicts of interest to disclose.

We got the agreement of the patient's parents to report this case.

\section{Acknowledgements}

We make our sincere acknowledgments for Doctor Trabelsi Madiha (genetic department in Charles Nicolle hospital of Tunis) and to the parents of our patient.

\section{References}

[1] Wiœniewska, M. and Mazurek, M. (2002) Trisomy 8 Mosaicism Syndrome. Journal of Applied Genetics, 43, 115-118.

[2] Fineman, R.M., Ablow, R.C., Howard, R.O., Albright, J. and Breg, W.R. (1975) Trisomy 8 Mosaicism Syndrome. Pediatrics, 56, 762-767.

[3] Agrawal, A. and Agrawal, R. (2011) Warkany Syndrome: A Rare Case Report. Case Reports in Pediatrics, 2011, Article ID: 437101. https://doi.org/10.1155/2011/437101

[4] Hale, N.E. and Keane, J.F. (2010) Piecing Together a Picture of Trisomy 8 Mosaicism Syndrome. The Journal of the American Osteopathic Association, 110, 21-23.

[5] Udayakumar, A.M. and Al-Kindy, A. (2013) Constitutional Trisomy 8 Mosaicism Syndrome: Case Report and Review. Journal of Pediatric Genetics, 2, 197-201.

[6] Giraldo, G., Gómez, A.M., Mora, L., Suarez-Obando, F. and Moreno, O. (2016) Mosaic Trisomy 8 Detected by Fibroblasts Cultured of Skin. Colombia Médica, 47, 100-104.

[7] Datta, A., Picker, J. and Rotenberg, A. (2010) Trisomy 8 Mosaicism and Favorable Outcome after Treatment of Infantile Spasms: Case Report. Journal of Child Neurology, 25, 1275-1277. https://doi.org/10.1177/0883073809357361 
[8] Engel, B.C., Podsakoff, G.M., Ireland, J.L., Smogorzewska, E.M., Carbonaro, D.A., Wilson, K., et al. (2007) Prolonged Pancytopenia in a Gene Therapy Patient with ADA-Deficient SCID and Trisomy 8 Mosaicism: A Case Report. Blood, 109, 503-506. https://doi.org/10.1182/blood-2006-06-031476

[9] Maserati, E., Aprili, F., Vinante, F., Locatelli, F., Amendola, G., Zatterale, A., et al. (2002) Trisomy 8 in Myelodysplasia and Acute Leukemia is Constitutional in 15 20\% of Cases. Genes Chromosomes Cancer, 33, 93-97.

https://doi.org/10.1002/gcc.1214

[10] van Haelst, M.M., Van Opstal, D., Lindhout, D. and Los, F.J. (2001) Management of Prenatally Detected Trisomy 8 Mosaicism. Prenatal Diagnosis, 21, 1075-1078. https://doi.org/10.1002/pd.215

[11] Cassina, M., Calò, A., Salviati, L., Alghisi, A., Montaldi, A. and Clementi, M. (2018) Prenatal Detection of Trisomy 8 Mosaicism: Pregnancy Outcome and Follow up of a Series of 17 Consecutive Cases. European Journal of Obstetrics \& Gynecology and Reproductive Biology, 221, 23-27. https://doi.org/10.1016/j.ejogrb.2017.12.012 